\title{
Bosonization in the Path Integral Formulation
}

\author{
Kazuo Fujikawa ${ }^{1}$ and Hiroshi Suzuki ${ }^{2}$ \\ 1 Mathematical Physics Laboratory, \\ RIKEN Nishina Center, Wako 351-0198, Japan \\ 2 Department of Physics, \\ Kyushu University, 6-10-1 Hakozaki, Higashi-ku, Fukuoka, 812-8581, Japan

\begin{abstract}
We establish the direct $d=2$ on-shell bosonization $\psi_{L}\left(x_{+}\right)=e^{i \xi\left(x_{+}\right)}$and $\psi_{R}^{\dagger}\left(x_{-}\right)=$ $e^{i \xi\left(x_{-}\right)}$in path integral formulation by deriving the off-shell relations $\psi_{L}(x) \psi_{R}^{\dagger}(x)=$ $\exp [i \xi(x)]$ and $\psi_{R}(x) \psi_{L}^{\dagger}(x)=\exp [-i \xi(x)]$. Similarly, the on-shell bosonization of the bosonic commuting spinor, $\phi_{L}\left(x_{+}\right)=i e^{-i \xi\left(x_{+}\right)} \partial^{+} e^{-i \chi\left(x_{+}\right)}, \phi_{R}^{\dagger}\left(x_{-}\right)=e^{-i \xi\left(x_{-}\right)-i \chi\left(x_{-}\right)}$ and $\phi_{R}\left(x_{-}\right)=i e^{i \xi\left(x_{-}\right)} \partial^{-} e^{+i \chi\left(x_{-}\right)}, \phi_{L}^{\dagger}\left(x_{+}\right)=e^{i \xi\left(x_{+}\right)+i \chi\left(x_{+}\right)}$, is established in path integral formulation by deriving the off-shell relations $\phi_{L}(x) \phi_{R}^{\dagger}(x)=i e^{-i \xi(x)} \partial^{+} e^{-i \chi(x)}$ and $\phi_{R}(x) \phi_{L}^{\dagger}(x)=i e^{i \xi(x)} \partial^{-} e^{i \chi(x)}$.
\end{abstract}

\section{Introduction}

It is known that the dynamical degrees of freedom of spinors are consistently described by the bosonic degrees of freedom in $d=2$ dimensional space-time [1, 2]. This fact, which is called the bosonization of spinors, has been analyzed in detail and it has been applied to various fields of theoretical physics such as conformal field theory and string theory and also to condensed matter physics [3, 4, 5, 6, 7, 8, 9, 10, 11]. The path integral formulation of bosonization, namely, a systematic derivation of bosonization rules in the framework of path integrals has been initiated at the early stage of this subject [12, 13, 14, 15, 16. The path integral bosonization has been applied to various problems [17]-[45], but, to our knowledge, the simplest bosonization rule in the form $\psi(x) \sim e^{i \xi(x)}$ appears to be missing. In this paper, we show how to realize this direct bosonization in path integral formulation.

We first recapitulate the known path integral bosonization rule

$$
\begin{aligned}
e^{i W\left(v_{\mu}\right)} & =\int \mathcal{D} \bar{\psi} \mathcal{D} \psi \exp \left\{i\left(\frac{1}{2 \pi}\right) \int d^{2} x\left[\bar{\psi} i \gamma^{\mu} \partial_{\mu} \psi+v_{\mu} \bar{\psi} \gamma^{\mu} \psi\right]\right\} \\
& =\int \mathcal{D} \xi \exp \left\{i\left(\frac{1}{4 \pi}\right) \int d^{2} x\left[\frac{1}{2} \partial^{\mu} \xi(x) \partial_{\mu} \xi(x)-2 v_{\mu} \epsilon^{\mu \nu} \partial_{\nu} \xi(x)\right]\right\},
\end{aligned}
$$

where the normalization of field variables is chosen to make the bosonization rule simpler. The basic ingredient to derive this path integral bosonization rule is the chiral Jacobian [46]

$$
\ln J(\beta)=\frac{i}{\pi} \int d^{2} x \beta(x)\left[\partial^{\mu} A_{\mu}+\frac{1}{2} \epsilon^{\mu \nu}\left(\partial_{\mu} V_{\nu}-\partial_{\nu} V_{\mu}\right)\right]
$$


for an infinitesimal chiral transformation $\psi(x) \rightarrow e^{i \beta(x) \gamma_{5}} \psi(x)$ and $\bar{\psi}(x) \rightarrow \bar{\psi}(x) e^{i \beta(x) \gamma_{5}}$ in the generic path integral

$$
\int \mathcal{D} \bar{\psi} \mathcal{D} \psi \exp \left\{i\left(\frac{1}{2 \pi}\right) \int d^{2} x\left[\bar{\psi} i \gamma^{\mu}\left(\partial_{\mu}-i V_{\mu}-i A_{\mu} \gamma_{5}\right) \psi\right]\right\} .
$$

To use this Jacobian factor, one may replace $v_{\mu}=\partial_{\mu} \alpha(x)+\epsilon_{\mu \nu} \partial^{\nu} \beta(x)$, which is valid in $d=$ 2, in the fermionic path integral in Eq. (11) and rewrite the fermionic Lagrangian as $\mathcal{L}=$ $\bar{\psi} i \gamma^{\mu}\left(\partial_{\mu}-i \partial_{\mu} \alpha(x)-i \gamma_{5} \partial_{\mu} \beta(x)\right) \psi$. The vector part $\partial_{\mu} \alpha(x)$ is transformed away by a suitable gauge transformation without generating any Jacobian factor. The axial-vector part $\partial_{\mu} \beta(x)$ is also transformed away by a suitable chiral gauge transformation but with a nontrivial Jacobian factor. The integrated form of the Jacobian is evaluated by the repeated applications of infinitesimal chiral transformations in the form $e^{-(i / 2 \pi) \int d^{2} x \partial_{\mu} \beta(x) \partial^{\mu} \beta(x)}$, and the final result is expressed in terms of $v_{\mu}(x)$ as an explicit generating functional. The final result is

$$
W\left(v_{\mu}\right)=\frac{1}{2 \pi} \int d^{2} x \epsilon^{\mu \nu} \partial_{\mu} v_{\nu}(x) \frac{1}{\partial_{\mu} \partial^{\mu}} \epsilon^{\alpha \beta} \partial_{\alpha} v_{\beta}(x),
$$

which is non-local with respect to $v_{\mu}(x)$, and it is also derived from the bosonic path integral in Eq. (11). See Refs. [12, 13, 14, 15] and the monograph [16] for further details.

The relation (11) shows that the theory of a free Dirac fermion $\psi$ and the theory of a free real Bose field $\xi$ define the identical generating functional $W\left(v_{\mu}\right)$ of connected Green's functions. The basic bosonization rule is thus

$$
\bar{\psi}(x) \gamma^{\mu} \psi(x)=-\epsilon^{\mu \nu} \partial_{\nu} \xi(x), \quad \text { or } \quad \bar{\psi}(x) \gamma^{\mu} \gamma_{5} \psi(x)=\partial^{\mu} \xi(x) .
$$

We used the relation $\gamma^{\mu} \gamma_{5}=-\epsilon^{\mu \nu} \gamma_{\nu}$ with $\epsilon^{10}=1$, and $\gamma^{0}=\sigma^{1}, \gamma^{1}=i \sigma^{2}, \gamma_{5}=\gamma^{1} \gamma^{0}=\sigma^{3}$. The notational convention is summarized in Appendix A. This current bosonization shows that the scalar field $\xi(x)$ is a pseudo-scalar. The above path integral bosonization (and its non-Abelian generalization) works for the known interesting examples in $d=2$ [16], for instance, the massless and massive Thirring models (the latter corresponds to the sine-Gordon model [1]) and the Wess-Zumino-Witten model [5].

\section{Bosonization of anti-commuting spinors}

It is interesting to examine if the direct bosonization of $\psi(x)$ in the form $\psi(x) \sim \exp [i \xi(x)][10$, 11] instead of the current in Eq. (5) is possible in path integral formulation by generalizing the formula (1). For this purpose, we start with Green's function defined by

$$
\square G(x-y)=\delta^{2}(x-y)
$$

which is best defined in Euclidean $d=2$ space and given by

$$
G_{E}(x-y)=\frac{1}{4 \pi} \ln \left[\left(x^{1}-y^{1}\right)^{2}+\left(x^{2}-y^{2}\right)^{2}\right]
$$


The corresponding Green's function in Minkowski metric is given by

$$
G_{M}(x-y)=\frac{-i}{4 \pi} \ln \left[\left(x^{1}-y^{1}\right)^{2}-\left(x^{0}-y^{0}\right)^{2}\right],
$$

where the imaginary factor $i$ arises from the rotation from Euclidean to Minkowski spaces by $x^{2} \rightarrow i x^{0}$, and thus $\int \square_{E} G_{E}\left(x^{1}-y^{1}, x^{2}-y^{2}\right) d x^{1} d x^{2}=\int(-1) \square_{M} G_{E}\left(x^{1}-y^{1}, i x^{0}-\right.$ $\left.i y^{0}\right) i d x^{1} d x^{0}=\int \square_{M} G_{M}\left(x^{1}-y^{1}, x^{0}-y^{0}\right) d x^{1} d x^{0}=1$. This expression (8) when combined with

$$
\begin{aligned}
\left(\frac{1}{2 \pi}\right) i \gamma^{\mu} \partial_{\mu}\left\langle T^{\star} \psi(x) \bar{\psi}(y)\right\rangle & =\int \mathcal{D} \bar{\psi} \mathcal{D} \psi\left\{-i\left(\frac{\delta}{\delta \bar{\psi}(x)} e^{i S}\right) \bar{\psi}(y)\right\} \\
& =i \delta_{M}^{2}(x-y)
\end{aligned}
$$

gives a correlation function of a free fermion

$$
\left\langle T^{\star} \psi(x) \bar{\psi}(y)\right\rangle=2 \pi \gamma^{\mu} \partial_{\mu} G_{M}(x-y)=-i \frac{\gamma^{\mu}(x-y)_{\mu}}{(x-y)^{2}} .
$$

In the present paper we often use the two components of a spinor $\psi(x)$, which are defined in our convention summarized in Appendix $\mathrm{A}$ by

$$
\psi(x)=\left(\begin{array}{c}
\psi_{R}(x) \\
\psi_{L}(x)
\end{array}\right), \quad \bar{\psi}(x)=\left(\begin{array}{c}
\psi_{L}^{\dagger}(x) \\
\psi_{R}^{\dagger}(x)
\end{array}\right),
$$

and the correlation function (10) is written as

$$
\left\langle T^{\star} \psi_{L}(x) \psi_{L}^{\dagger}(y)\right\rangle=\frac{1}{i(x-y)_{+}}, \quad\left\langle T^{\star} \psi_{R}(x) \psi_{R}^{\dagger}(y)\right\rangle=\frac{1}{i(x-y)_{-}} .
$$

We note that $x_{ \pm}=x_{0} \pm x_{1}$ and $x^{2}=x_{+} x_{-}$in this convention, and the free Dirac action is written as

$$
S=\int d^{2} x \bar{\psi} i \gamma^{\mu} \partial_{\mu} \psi=\int d^{2} x\left[\psi_{R}^{\dagger} i \partial_{-} \psi_{R}+\psi_{L}^{\dagger} i \partial_{+} \psi_{L}\right] .
$$

As for the bosonic variable $\xi(x)$, we have

$$
\begin{aligned}
\left(\frac{1}{4 \pi}\right) \partial_{\mu} \partial^{\mu}\left\langle T^{\star} \xi(x) \xi(y)\right\rangle & =\int \mathcal{D} \xi\left(\frac{1}{4 \pi}\right) \partial_{\mu} \partial^{\mu} \xi(x) \xi(y) \exp \left\{i \int d^{2} x\left[\left(\frac{1}{4 \pi}\right) \frac{1}{2} \partial^{\mu} \xi \partial_{\mu} \xi\right]\right\} \\
& =\int \mathcal{D} \xi\left[i \frac{\delta e^{i S}}{\delta \xi(x)}\right] \xi(y) \\
& =-i \delta_{M}^{2}(x-y)
\end{aligned}
$$

which implies

$$
\left\langle T^{\star} \xi(x) \xi(y)\right\rangle=-i 4 \pi G_{M}(x-y)=-\ln \left[\left(x^{1}-y^{1}\right)^{2}-\left(x^{0}-y^{0}\right)^{2}\right],
$$


and thus

$$
\begin{aligned}
\left\langle T^{\star} \exp [i \xi(x)] \exp [-i \xi(y)]\right\rangle & =\Lambda^{-2} \exp \left\{-\ln \left[-(x-y)^{2}\right]\right\} \\
& =\Lambda^{-2} \frac{1}{\left[-(x-y)_{+}(x-y)_{-}\right]}
\end{aligned}
$$

where we denote the divergence at coincident points by $\Lambda$. This last correlation function or more generally the correlation function in the presence of the composite operator $\exp [i \xi(x)] \exp [-i \xi(y)]$ is evaluated in path integral by adding a source term

$$
\begin{aligned}
& \int \mathcal{D} \xi \exp \left\{i \int d^{2} x\left[\left(\frac{1}{4 \pi}\right) \frac{1}{2} \partial^{\mu} \xi \partial_{\mu} \xi+\xi(x) J(x)\right]\right\} \\
& =\int \mathcal{D} \xi \exp \left\{i \int d^{2} x\left[\left(\frac{1}{4 \pi}\right) \frac{1}{2} \partial^{\mu} \xi \partial_{\mu} \xi\right]\right\} \exp \left[\frac{i}{2} \int d^{2} u d^{2} v J(u) 4 \pi G_{M}(u-v) J(v)\right]
\end{aligned}
$$

and replacing $J(u) \rightarrow J(u)+\delta(u-x)-\delta(u-y)$. We thus have

$$
T^{\star} \Lambda^{2} \exp [i \xi(x)] \exp [-i \xi(y)]=\Lambda^{2}\left\langle T^{\star} \exp [i \xi(x)] \exp [-i \xi(y)]\right\rangle: \exp [i \xi(x)-i \xi(y)]:
$$

where normal ordering means no more contraction between $\xi(x)$ and $\xi(y)$, and those variables are contracted with variables contained in $\xi(x) J(x)$ outside the composite operator in path integral.

We now recall an important formula that is shown using Cauchy's lemma, of which proof is given in Appendix B for completeness,

$$
\begin{aligned}
& \int \mathcal{D} \bar{\psi} \mathcal{D} \psi \exp \left\{i\left(\frac{1}{2 \pi}\right) \int d^{2} x\left[\bar{\psi} i \gamma^{\mu} \partial_{\mu} \psi\right]\right\} \prod_{j=1}^{N} \prod_{k=1}^{N} \psi_{L}\left(x_{j}\right) \psi_{R}^{\dagger}\left(x_{j}\right) \psi_{R}\left(y_{k}\right) \psi_{L}^{\dagger}\left(y_{k}\right) \\
& =\operatorname{det} \frac{1}{i\left(x_{i}-y_{k}\right)_{+}} \operatorname{det} \frac{1}{i\left(x_{i}-y_{k}\right)_{-}} \\
& =\prod_{j=1}^{N} \prod_{k=1}^{N} \frac{1}{\left[-\left(x_{j}-y_{k}\right)^{2}\right]} \prod_{j_{1}>j_{2}}^{N} \prod_{k_{1}>k_{2}}^{N}\left[-\left(x_{j_{1}}-x_{j_{2}}\right)^{2}\right]\left[-\left(y_{k_{1}}-y_{k_{2}}\right)^{2}\right] \\
& =\int \mathcal{D} \xi \exp \left\{i\left(\frac{1}{4 \pi}\right) \int d^{2} x\left[\frac{1}{2} \partial^{\mu} \xi(x) \partial_{\mu} \xi(x)\right]\right\} \prod_{j=1}^{N} \prod_{k=1}^{N} \Lambda \exp \left[i \xi\left(x_{j}\right)\right] \Lambda \exp \left[-i \xi\left(y_{k}\right)\right] .
\end{aligned}
$$

The importance of Cauchy's lemma in bosonization has been noted in Ref. [11]. We then 
establish

$$
\begin{aligned}
& e^{i W\left(v_{\mu}, j_{R}, j_{L}\right)} \\
& =\int \mathcal{D} \bar{\psi} \mathcal{D} \psi \exp \left\{i\left(\frac{1}{2 \pi}\right) \int d^{2} x\left[\bar{\psi} i \gamma^{\mu} \partial_{\mu} \psi+v_{\mu} \bar{\psi} \gamma^{\mu} \psi-j_{L}(x) \psi_{L} \psi_{R}^{\dagger}-j_{R}(x) \psi_{R} \psi_{L}^{\dagger}\right]\right\} \\
& =\int \mathcal{D} \bar{\psi} \mathcal{D} \psi \exp \left\{i\left(\frac{1}{2 \pi}\right) \int d^{2} x\right. \\
& \left.\quad \times\left[\bar{\psi} i \gamma^{\mu} \partial_{\mu} \psi-j_{L}(x) e^{-2 i \beta} \psi_{L} \psi_{R}^{\dagger}-j_{R}(x) e^{2 i \beta} \psi_{R} \psi_{L}^{\dagger}-\partial_{\mu} \beta \partial^{\mu} \beta\right]\right\} \\
& =\int \mathcal{D} \xi \exp \left\{i\left(\frac{1}{4 \pi}\right) \int d^{2} x\right. \\
& \left.\quad \times\left[\frac{1}{2} \partial^{\mu} \xi(x) \partial_{\mu} \xi(x)-2 j_{L}(x) \Lambda e^{-2 i \beta+i \xi}-2 j_{R}(x) \Lambda e^{2 i \beta-i \xi}-2 \partial_{\mu} \beta \partial^{\mu} \beta\right]\right\} \\
& =\int \mathcal{D} \xi \exp \left\{i\left(\frac{1}{4 \pi}\right) \int d^{2} x\left[\frac{1}{2} \partial^{\mu} \xi(x) \partial_{\mu} \xi(x)-2 v_{\mu} \epsilon^{\mu \nu} \partial_{\nu} \xi(x)-2 j_{L} \Lambda e^{i \xi}-2 j_{R} \Lambda e^{-i \xi}\right]\right\},
\end{aligned}
$$

where in the second line, we replaced $v_{\mu}=\partial_{\mu} \alpha(x)+\epsilon_{\mu \nu} \partial^{\nu} \beta(x)$ and the fermion Lagrangian is changed to $\mathcal{L}=\bar{\psi} i \gamma^{\mu}\left(\partial_{\mu}-i \partial_{\mu} \alpha(x)-i \gamma_{5} \partial_{\mu} \beta(x)\right) \psi$. The vector freedom $\partial_{\mu} \alpha(x)$ is gauge transformed away without modifying the rest of the terms and without any Jacobian. The axial vector freedom $\partial_{\mu} \beta(x)$ is transformed away by the change of the fermion variables $\psi \rightarrow e^{i \beta \gamma_{5}} \psi$ and $\bar{\psi} \rightarrow \bar{\psi} e^{i \beta \gamma_{5}}$, which give rise to an integrated Jacobian factor $-i /(2 \pi) \int d^{2} x \partial_{\mu} \beta \partial^{\mu} \beta$ [12, 13, 14, 15] together with the modifications of the rest of the terms; this integrated Jacobian is confirmed to be correct by considering an infinitesimal variation of $\beta$ and obtaining $(i / \pi) \int d^{2} x \delta \beta \partial_{\mu} \partial^{\mu} \beta$.

We then expand the fermionic path integral in powers of $j_{L}$ and $j_{R}$ in the second line in Eq. (20), which is then converted to a bosonic path integral using the formula (19). The final result is then re-summed to an exponential form in bosonic path integral. In the last step in Eq. (20), we changed the path integral variable $\xi-2 \beta \rightarrow \xi$ by noting $\mathcal{D}(\xi-2 \beta)=\mathcal{D} \xi$ and used the relation $\partial_{\nu} \partial^{\nu} \beta=-\partial_{\nu} v_{\mu} \epsilon^{\mu \nu}$.

We have thus established path integral bosonization rules in Eq. (20),

$$
\bar{\psi} \gamma^{\mu} \gamma_{5} \psi=\partial^{\mu} \xi, \quad \psi_{L}(x) \psi_{R}^{\dagger}(x)=\Lambda \exp [i \xi(x)], \quad \psi_{R}(x) \psi_{L}^{\dagger}(x)=\Lambda \exp [-i \xi(x)]
$$

where the first relation is also written as

$$
\psi_{L}^{\dagger}(x) \psi_{L}(x)=-\partial^{+} \xi(x), \quad \psi_{R}^{\dagger}(x) \psi_{R}(x)=\partial^{-} \xi(x) .
$$

The consistency of the last two relations in Eq. (21) with the first relation is confirmed by comparing (the short distance expansions)

$$
\begin{aligned}
& T^{\star} \psi_{L}(x) \psi_{R}^{\dagger}(x) \psi_{R}(y) \psi_{L}^{\dagger}(y) \\
& =\frac{1}{i(x-y)_{+}} \frac{1}{i(x-y)_{-}}+\frac{1}{i(x-y)_{+}} \psi_{R}^{\dagger}(y) \psi_{R}(y)-\frac{1}{i(x-y)_{-}} \psi_{L}^{\dagger}(y) \psi_{L}(y)+\text { regular terms, }
\end{aligned}
$$


which is established in the path integral,

$$
\begin{aligned}
& \int \mathcal{D} \bar{\psi} \mathcal{D} \psi \psi_{L}(x) \psi_{R}^{\dagger}(x) \psi_{R}(y) \psi_{L}^{\dagger}(y) \exp \left\{i\left(\frac{1}{2 \pi}\right) \int d^{2} x\left[\bar{\psi} i \gamma^{\mu} \partial_{\mu} \psi\right]\right\} \\
& \quad \times \exp \left\{\int d^{2} x\left[\eta_{L}^{\dagger}(x) \psi_{L}(x)+\psi_{L}^{\dagger}(y) \eta_{L}(x)+\eta_{R}^{\dagger}(x) \psi_{R}(x)+\psi_{R}^{\dagger}(x) \eta_{R}(x)\right]\right\}
\end{aligned}
$$

by expanding in powers of source functions $\eta(x)$, with the bosonic relation derived from Eq. (18),

$$
\begin{aligned}
& T^{\star} \Lambda^{2} \exp [i \xi(x)] \exp [-i \xi(y)] \\
& =\frac{1}{i(x-y)_{+}} \frac{1}{i(x-y)_{-}}\left\{1+i(x-y)_{+} \partial^{+} \xi(y)+i(x-y)_{-} \partial^{-} \xi(y)\right\}+\text { regular terms }
\end{aligned}
$$

where regular terms mean no singularities for $O\left((x-y)_{+}\right)=O\left((x-y)_{-}\right)$. These two expansions (23) and (25) are consistent with the relations in Eq. (22).

These bosonization rules (21) are consistent with the parity transformation $\psi^{\prime}\left(x^{\prime}\right)=$ $\gamma^{0} \psi(x)$ and $\xi^{\prime}\left(x^{\prime}\right)=-\xi(x)$ with $x^{\prime}=\left(x^{0},-x^{1}\right)$, and Lorentz properties

$$
\begin{gathered}
i \bar{\psi}(x) \gamma_{5} \psi(x)=i \psi_{L}^{\dagger}(x) \psi_{R}(x)-i \psi_{R}^{\dagger}(x) \psi_{L}(x)=-2 \Lambda \sin \xi(x), \\
\bar{\psi}(x) \psi(x)=\psi_{L}^{\dagger}(x) \psi_{R}(x)+\psi_{R}^{\dagger}(x) \psi_{L}(x)=-2 \Lambda \cos \xi(x) .
\end{gathered}
$$

They are also consistent with chiral Ward-Takahashi identities in path integral

$$
\begin{aligned}
& i \partial_{\mu}\left\langle T^{\star}\left(\frac{1}{2 \pi}\right) \bar{\psi}(x) \gamma^{\mu} \gamma_{5} \psi(x) \prod_{j=1}^{N} \prod_{k=1}^{N} \psi_{L}\left(x_{j}\right) \psi_{R}^{\dagger}\left(x_{j}\right) \psi_{R}\left(y_{k}\right) \psi_{L}^{\dagger}\left(y_{k}\right)\right\rangle \\
& =\sum_{j} 2 i \delta^{2}\left(x-x_{j}\right)\left\langle T^{\star} \prod_{j=1}^{N} \prod_{k=1}^{N} \psi_{L}\left(x_{j}\right) \psi_{R}^{\dagger}\left(x_{j}\right) \psi_{R}\left(y_{k}\right) \psi_{L}^{\dagger}\left(y_{k}\right)\right\rangle \\
& \quad-\sum_{k} 2 i \delta^{2}\left(x-y_{k}\right)\left\langle T^{\star} \prod_{j=1}^{N} \prod_{k=1}^{N} \psi_{L}\left(x_{j}\right) \psi_{R}^{\dagger}\left(x_{j}\right) \psi_{R}\left(y_{k}\right) \psi_{L}^{\dagger}\left(y_{k}\right)\right\rangle
\end{aligned}
$$

and

$$
\begin{aligned}
& i \partial_{\mu}\left\langle T^{\star}\left(\frac{1}{2 \pi}\right) \partial^{\mu} \xi(x) \prod_{j=1}^{N} \prod_{k=1}^{N} \Lambda \exp \left[i \xi\left(x_{j}\right)\right] \Lambda \exp \left[-i \xi\left(y_{k}\right)\right]\right\rangle \\
& =\sum_{j} 2 i \delta^{2}\left(x-x_{j}\right)\left\langle T^{\star} \prod_{j=1}^{N} \prod_{k=1}^{N} \Lambda \exp \left[i \xi\left(x_{j}\right)\right] \Lambda \exp \left[-i \xi\left(y_{k}\right)\right]\right\rangle \\
& \quad-\sum_{k} 2 i \delta^{2}\left(x-y_{k}\right)\left\langle T^{\star} \prod_{j=1}^{N} \prod_{k=1}^{N} \Lambda \exp \left[i \xi\left(x_{j}\right)\right] \Lambda \exp \left[-i \xi\left(y_{k}\right)\right]\right\rangle
\end{aligned}
$$

where the bosonic form of identities are obtained by considering the change of integration variable $\xi(x) \rightarrow \xi(x)^{\prime}=\xi(x)+\alpha(x)$ in the bosonic path integral. Equivalently, the current algebra in operator formalism

$$
i\left[\left(\frac{1}{2 \pi}\right) \bar{\psi}(x) \gamma^{0} \gamma_{5} \psi(x), \psi_{L}(y) \psi_{R}^{\dagger}(y)\right] \delta\left(x^{0}-y^{0}\right)=2 i \psi_{L}(y) \psi_{R}^{\dagger}(y) \delta^{2}(x-y),
$$


for example, is consistently realized by

$$
i\left[\left(\frac{1}{2 \pi}\right) \partial^{0} \xi(x), \Lambda e^{i \xi(y)}\right] \delta\left(x^{0}-y^{0}\right)=2 i \Lambda e^{i \xi(y)} \delta^{2}(x-y),
$$

since the canonical conjugate of $\xi(x)$ is $\Pi(x)=1 /(4 \pi) \partial^{0} \xi(x)$.

One may thus identify the last two relations in Eq. (21) as the path integral version of the off-shell direct bosonization rules. In the limit of on-shell fields with $\partial^{+} \partial^{-} \xi(x)=0$ and $\xi(x)=\xi\left(x_{+}\right)+\xi\left(x_{-}\right)$, these direct bosonization rules imply

$$
\psi_{L}\left(x_{+}\right)=\Lambda^{1 / 2} e^{i \xi\left(x_{+}\right)}=: e^{i \xi\left(x_{+}\right)}:, \quad \psi_{R}^{\dagger}\left(x_{-}\right)=\Lambda^{1 / 2} e^{i \xi\left(x_{-}\right)}=: e^{i \xi\left(x_{-}\right)}:,
$$

which is the familiar direct bosonization formula in operator formalism [10, 11]. Starting with the second relation of Eq. (21), which is valid for general off-shell fields, we obtain this particular form of decomposition by considering a subset of $\psi_{L}(x)$ and $\psi_{R}^{\dagger}(x)$ which satisfy the equations of motion $\partial^{-} \psi_{L}(x)=0$ and $\partial^{+} \psi_{R}^{\dagger}(x)=0$ and thus depend only on $x_{+}$ and $x_{-}$, respectively. The factor $\Lambda^{1 / 2}$ is absorbed by normal ordering. The formula (19) is also consistent with the factorization into $x_{+}$and $x_{-}$sectors in the last two relations in Eq. (21). Note the notational conventions $x_{ \pm}=x_{0} \pm x_{1}, x^{2}=x_{+} x_{-}$, and $\partial^{ \pm} x_{ \pm}=1$.

We emphasize that we cannot define the path integral in terms of the on-shell variables $\xi\left(x_{+}\right)$and $\xi\left(x_{-}\right)$directly, unlike the operator formalism such as conformal field theory where $\xi\left(x_{+}\right)$and $\xi\left(x_{-}\right)$are treated as independent variables.

\section{Bosonization of commuting spinors}

The bosonic spinors, namely, commuting spinors appear in the quantization of the spinning string [ [, 9]. The Pauli-Villars regularization is also implemented in path integrals using those bosonic fermions [16].

We here discuss the bosonization of commuting spinors in $d=2$. We can readily establish the relation

$$
\begin{aligned}
e^{i W\left(v_{\mu}\right)} & =\int \mathcal{D} \bar{\phi} \mathcal{D} \phi \exp \left\{i\left(\frac{1}{2 \pi}\right) \int d^{2} x\left[\bar{\phi} i \gamma^{\mu} \partial_{\mu} \phi+v_{\mu} \bar{\phi} \gamma^{\mu} \phi\right]\right\} \\
& =\int \mathcal{D} \xi \exp \left\{i\left(\frac{1}{4 \pi}\right) \int d^{2} x\left[-\frac{1}{2} \partial^{\mu} \xi(x) \partial_{\mu} \xi(x)-2 v_{\mu} \epsilon^{\mu \nu} \partial_{\nu} \xi(x)\right]\right\}
\end{aligned}
$$

where $\phi(x)$ stands for the commuting spinor in $d=2$. The basic difference of this expression from the previous one (1) is that the signature in front of the action for the bosonic field $\xi(x)$ differs, namely, we have - instead of + . This minus sign must be adopted since the Jacobian changes signature for the commuting variables $\phi$ from the anticommuting Grassmann variables $\psi$. The boson field $\xi(x)$ in the present case corresponds to a negative normed field.

The bosonization rule of the current

$$
\bar{\phi} \gamma^{\mu} \phi=-\epsilon^{\mu \nu} \partial_{\nu} \xi(x)
$$


is the same as before

$$
\phi_{L}^{\dagger}(x) \phi_{L}(x)=-\partial^{+} \xi(x), \quad \phi_{R}^{\dagger}(x) \phi_{R}(x)=\partial^{-} \xi(x) .
$$

But we have

$$
\left\langle T^{\star} \xi(x) \xi(y)\right\rangle=i 4 \pi G_{M}(x-y)=\ln \left[\left(x^{1}-y^{1}\right)^{2}-\left(x^{0}-y^{0}\right)^{2}\right],
$$

and thus

$$
\begin{aligned}
\left\langle T^{\star} \exp [i \xi(x)] \exp [-i \xi(y)]\right\rangle & =\Lambda^{2} \exp \left[i 4 \pi G_{M}(x-y)\right] \\
& =\Lambda^{2}\left[-(x-y)^{2}\right]
\end{aligned}
$$

which cannot reproduce the fermion poles.

However, the relation

$$
(-1)\left\langle T^{\star} \phi_{L}(x) \phi_{R}^{\dagger}(x) \phi_{R}(y) \phi_{L}^{\dagger}(y)\right\rangle=\left[\frac{1}{i(x-y)_{+}}\right]\left[\frac{1}{i(x-y)_{-}}\right],
$$

is established by noting

$$
\left\langle T^{\star} \phi_{R}(x) \phi_{R}^{\dagger}(y)\right\rangle=\frac{1}{i(x-y)_{-}}, \quad\left\langle T^{\star} \phi_{L}(x) \phi_{L}^{\dagger}(y)\right\rangle=\frac{1}{i(x-y)_{+}} .
$$

This may be compared with

$$
\left\langle T^{\star} \exp [-i \xi(x)] \partial^{+} \exp [-i \chi(x)] \exp [i \xi(y)] \partial^{-} \exp [i \chi(y)]\right\rangle=\left[\frac{1}{i(x-y)_{+}}\right]\left[\frac{1}{i(x-y)_{-}}\right]
$$

using the positive normed auxiliary (pseudo-scalar) field $\chi(x)$,

$$
\left\langle T^{\star} \Lambda^{2} \exp [i \chi(x)] \exp [-i \chi(y)]\right\rangle=\frac{1}{\left[-(x-y)^{2}\right]},
$$

with $\left\langle T^{\star} \chi(x) \chi(y)\right\rangle=-\ln \left[\left(x^{1}-y^{1}\right)^{2}-\left(x^{0}-y^{0}\right)^{2}\right]$; the role of $\chi(x)$ is to provide a factor $1 /\left[-(x-y)^{2}\right]^{2}$ without spoiling the bosonization rule of the current in Eq. (33). Note that $\partial^{ \pm} x_{ \pm}=1$ and $\partial^{ \pm} x_{\mp}=0$.

We confirm that the extra field $\chi$, which has been used in the conformal field theory approach [8, 9] but was introduced in a somewhat ad hoc manner in the present study, does not spoil the basic bosonization of the current in Eq. (33). We check this by examining the short distance expansion, which tests algebraic properties,

$$
\begin{aligned}
& (-1) T^{\star} \phi_{L}(x) \phi_{R}^{\dagger}(x) \phi_{R}(y) \phi_{L}^{\dagger}(y) \\
& =\left[\frac{1}{i(x-y)_{+}}\right]\left[\frac{1}{i(x-y)_{-}}\right]+\left[\frac{1}{i(x-y)_{-}}\right] \phi_{L}(y) \phi_{L}^{\dagger}(y)-\left[\frac{1}{i(x-y)_{+}}\right] \phi_{R}(y) \phi_{R}^{\dagger}(y) \\
& \quad+\text { regular terms, }
\end{aligned}
$$


which may be compared with (by using a relation analogous to Eq. (18))

$$
\begin{aligned}
T^{\star} & \exp [-i \xi(x)] \partial^{+} \exp [-i \chi(x)] \exp [i \xi(y)] \partial^{-} \exp [i \chi(y)] \\
= & \frac{1}{\left[-(x-y)_{+}(x-y)_{-}\right]}\left\{1-i(x-y)_{+} \partial^{+} \xi(y)-i(x-y)_{-} \partial^{-} \xi(y)+\cdots\right\} \\
= & {\left[\frac{1}{i(x-y)_{+}}\right]\left[\frac{1}{i(x-y)_{-}}\right]-\left[\frac{1}{i(x-y)_{-}}\right] \partial^{+} \xi(y)-\left[\frac{1}{i(x-y)_{+}}\right] \partial^{-} \xi(y) } \\
\quad & \quad+\text { regular terms, }
\end{aligned}
$$

where regular terms mean no singularities for $O\left((x-y)_{+}\right)=O\left((x-y)_{-}\right)$. These expansions (41) and (42) are consistent with Eq. (34).

We thus expect the off-shell bosonization rules

$$
\phi_{L}(x) \phi_{R}^{\dagger}(x)=i e^{-i \xi(x)} \partial^{+} e^{-i \chi(x)}, \quad \phi_{R}(x) \phi_{L}^{\dagger}(x)=i e^{i \xi(x)} \partial^{-} e^{i \chi(x)},
$$

which are consistent with parity transformation. We can in fact establish these rules together with Eq. (33) by repeating the same steps as in Eq. (20),

$$
\begin{aligned}
& e^{i W\left(v_{\mu}, j_{R}, j_{L}\right)} \int \mathcal{D} \bar{\phi} \mathcal{D} \phi \exp \left\{i\left(\frac{1}{2 \pi}\right) \int d^{2} x\left[\bar{\phi} i \gamma^{\mu} \partial_{\mu} \phi+v_{\mu}(x) \bar{\phi} \gamma^{\mu} \phi-j_{L}(x) \phi_{L} \phi_{R}^{\dagger}-j_{R}(x) \phi_{R} \phi_{L}^{\dagger}\right]\right\} \\
&= \int \mathcal{D} \bar{\phi} \mathcal{D} \phi \exp \left\{i\left(\frac{1}{2 \pi}\right) \int d^{2} x\right. \\
&\left.\times\left[\bar{\phi} i \gamma^{\mu} \partial_{\mu} \phi-j_{L}(x) e^{-2 i \beta} \phi_{L} \phi_{R}^{\dagger}-j_{R}(x) e^{2 i \beta} \phi_{R} \phi_{L}^{\dagger}+\partial_{\mu} \beta \partial^{\mu} \beta\right]\right\} \\
&= \int \mathcal{D} \xi \mathcal{D} \chi \exp \left\{i ( \frac { 1 } { 4 \pi } ) \int d ^ { 2 } x \left[-\frac{1}{2} \partial^{\mu} \xi(x) \partial_{\mu} \xi(x)+\frac{1}{2} \partial^{\mu} \chi(x) \partial_{\mu} \chi(x)\right.\right. \\
&=\int \mathcal{D} \xi \mathcal{D} \chi \exp \left\{i ( \frac { 1 } { 4 \pi } ) \int d ^ { 2 } x \left[-\frac{1}{2} \partial^{\mu} \xi(x) \partial_{\mu} \xi(x)+\frac{1}{2} \partial^{\mu} \chi(x) \partial_{\mu} \chi(x)-2 v_{\mu}(x) \epsilon^{\mu \nu} \partial_{\nu} \xi(x)\right.\right. \\
&\left.\left.=2 j_{L}(x) i e^{-2 i \beta-i \xi} \partial^{+} e^{-i \chi}-2 j_{R}(x) i e^{2 i \beta+i \xi} \partial^{-} e^{i \chi}+2 \partial_{\mu} \beta \partial^{\mu} \beta\right]\right\}
\end{aligned}
$$

where in the second line, we replaced $v_{\mu}=\partial_{\mu} \alpha(x)+\epsilon_{\mu \nu} \partial^{\nu} \beta(x)$ and eliminated $\partial_{\mu} \alpha$ and $\partial^{\nu} \beta$ by suitable gauge transformations. Note the sign change of the Jacobian factor $\partial_{\mu} \beta \partial^{\mu} \beta$. 
In the second line, we used a bosonic-spinor analogue of Cauchy's lemma in Eq. (19),

$$
\begin{gathered}
\int \mathcal{D} \bar{\phi} \mathcal{D} \phi \exp \left\{i\left(\frac{1}{2 \pi}\right) \int d^{2} x\left[\bar{\phi} i \gamma^{\mu} \partial_{\mu} \phi\right]\right\} \prod_{j=1}^{N} \prod_{k=1}^{N} \phi_{L}\left(x_{j}\right) \phi_{R}^{\dagger}\left(x_{j}\right) \phi_{R}\left(y_{k}\right) \phi_{L}^{\dagger}\left(y_{k}\right) \\
=\left[\sum_{\left\{k_{1}, \ldots, k_{N}\right\}} \frac{1}{i\left(x_{1}-y_{k_{1}}\right)_{+}} \frac{1}{i\left(x_{2}-y_{k_{2}}\right)_{+}} \cdots \frac{1}{i\left(x_{N}-y_{k_{N}}\right)_{+}}\right] \\
\times\left[\sum_{\left\{k_{1}, \ldots, k_{N}\right\}} \frac{1}{i\left(y_{1}-x_{k_{1}}\right)_{-}} \frac{1}{i\left(y_{2}-x_{k_{2}}\right)_{-}} \cdots \frac{1}{i\left(y_{N}-x_{\left.k_{N}\right)_{-}}\right.}\right] \\
=\int \mathcal{D} \xi \mathcal{D} \chi \exp \left\{i\left(\frac{1}{4 \pi}\right) \int d^{2} x\left[-\frac{1}{2} \partial^{\mu} \xi(x) \partial_{\mu} \xi(x)+\frac{1}{2} \partial^{\mu} \chi(x) \partial_{\mu} \chi(x)\right]\right\} \\
\times \prod_{j=1}^{N} \prod_{k=1}^{N}\left[i e^{-i \xi\left(x_{j}\right)} \partial^{+} e^{-i \chi\left(x_{j}\right)}\right]\left[i e^{i \xi\left(y_{k}\right)} \partial^{-} e^{i \chi\left(y_{k}\right)}\right],
\end{gathered}
$$

where the summation runs over all the permutations of $(1,2, \ldots, N)$. The proof of this formula is given in Appendix C. In the last step in Eq. (44), we changed the integration variable $\xi+2 \beta \rightarrow \xi$.

For on-shell fields with $\xi(x)=\xi\left(x_{+}\right)+\xi\left(x_{-}\right)$and $\chi(x)=\chi\left(x_{+}\right)+\chi\left(x_{-}\right)$, the relations in Eq. (43) imply

$$
\begin{array}{llrl}
\phi_{L}\left(x_{+}\right) & =i e^{-i \xi\left(x_{+}\right)} \partial^{+} e^{-i \chi\left(x_{+}\right)}, & \phi_{R}^{\dagger}\left(x_{-}\right) & =e^{-i \xi\left(x_{-}\right)-i \chi\left(x_{-}\right)} \\
\phi_{R}\left(x_{-}\right) & =i e^{i \xi\left(x_{-}\right)} \partial^{-} e^{+i \chi\left(x_{-}\right)}, & \phi_{L}^{\dagger}\left(x_{+}\right) & =e^{i \xi\left(x_{+}\right)+i \chi\left(x_{+}\right)}
\end{array}
$$

Here, we have noted that $\phi_{L}(x)$ and $\phi_{R}^{\dagger}(x)$ depend only on $x_{+}$and $x_{-}$, respectively, if one considers a subset of $\phi_{L}(x)$ and $\phi_{R}^{\dagger}(x)$ which satisfy the equations of motion $\partial^{-} \phi_{L}(x)=0$ and $\partial^{+} \phi_{R}^{\dagger}(x)=0$. The parity symmetry is preserved in these formulas, but the hermiticity such as $\left(\phi_{L}\left(x_{+}\right)\right)^{\dagger}=\phi_{L}^{\dagger}\left(x_{+}\right)$is lost. In Euclidean formulation such as in conformal field theory, one may treat $\phi_{L}\left(x_{+}\right)$and $\phi_{L}^{\dagger}\left(x_{+}\right)$as independent quantities and thus this formulation may be used; in fact, this trick has been used for the commuting ghosts in spinning string theory where $\phi_{L}\left(x_{+}\right) \rightarrow-i \beta$ and $\phi_{L}^{\dagger}\left(x_{+}\right) \rightarrow \gamma$, and thus these two fields are literally independent [8, 9]. To be precise, after the Wick rotation $x_{+} \rightarrow-z$ and $x_{-} \rightarrow \bar{z}$, we find the correspondence (after a suitable re-definition of field variables),

$$
\begin{aligned}
\beta(z) & =e^{-\phi(z)+\chi(z)} \partial_{z} \chi(z), & \gamma(z) & =e^{\phi(z)-\chi(z)}, \\
\bar{\beta}(\bar{z}) & =e^{-\bar{\phi}(\bar{z})+\bar{\chi}(\bar{z})} \partial_{\bar{z}} \bar{\chi}(\bar{z}), & \bar{\gamma}(\bar{z}) & =e^{\bar{\phi}(\bar{z})-\bar{\chi}(\bar{z})} .
\end{aligned}
$$

See, for example, Section 10.4 in Ref. [8].

\section{Discussion}

We have shown that the direct on-shell bosonization such as $\psi_{L}\left(x_{+}\right)=e^{i \xi\left(x_{+}\right)}$and $\psi_{R}^{\dagger}\left(x_{-}\right)=$ $e^{i \xi\left(x_{-}\right)}$in $d=2$ is understood in a systematic way by path integral formulation which is 
based on off-shell fields. This fact must be conceptually satisfactory when one considers the path integral bosonization. The bosonization itself has many applications in mathematical physics such as conformal field theory and also in condensed matter physics.

Finally, we mention the first quantization of string theory briefly, where the bosonization of Faddeev-Popov ghosts historically played important roles [47]. For bosonic critical string theory, the anti-commuting reparameterization ghosts are described by a formal Dirac Lagrangian in $d=2$ Euclidean space [48]

$$
\int \mathcal{D} \xi \mathcal{D} \eta \exp \left\{\left(\frac{1}{2 \pi}\right) \int d^{2} x \xi(x)\left(\sigma_{1} \partial_{1}+\sigma_{3} \partial_{2}\right) \eta(x)\right\},
$$

where

$$
\xi(x)=\left(\begin{array}{l}
\xi_{1} \\
\xi_{2}
\end{array}\right), \quad \eta(x)=\left(\begin{array}{l}
\eta^{1} \\
\eta^{2}
\end{array}\right)
$$

and $\eta$ stands for the reparameterization ghosts and $\xi(x)$ for the multiplier fields. Our bosonization of anti-commuting spinors is directly applied to this case, and the result gives rise to the formula in the modern notation of $b c$ ghosts [8, 9]. See also Refs. [49, 50]. For spinning critical string theory, the path integral for the commuting ghosts associated with the fixing of supersymmetry transformation of gravitino is described by a formal Dirac Lagrangian in $d=2$ Euclidean space [51, 52]

$$
\int \mathcal{D} \bar{C} \mathcal{D} C \exp \left\{\frac{1}{2 \pi} \int d^{2} x \bar{C}(x)\left(\sigma_{1} \partial_{1}-\sigma_{2} \partial_{2}\right) C(x)\right\},
$$

where $C(x)$ stands for commuting spinor ghosts and $\bar{C}(x)$ for the multiplier fields. This is bosonized using the above result of commuting spinors, and the result agrees with the bosonization in the modern notation of $\beta \gamma$ ghosts [8, 9]. In these primitive notations (48) and (50), the bosonization of two component spinors appears in a more explicit manner.

\section{A Notational convention}

We here summarize the notations in 2-dimensional Minkowski space-time which is defined by

$$
\left\{\gamma^{\mu}, \gamma^{\nu}\right\}=2 g^{\mu \nu}
$$

with $g_{\mu \nu}=(1,-1)=g^{\mu \nu}$ and $\epsilon^{10}=1$. We define $\gamma^{0}=\sigma^{1}, \gamma^{1}=i \sigma^{2}, \gamma_{5}=\gamma^{1} \gamma^{0}=\sigma^{3}$ with $\gamma^{\mu} \gamma_{5}=-\epsilon^{\mu \nu} \gamma_{\nu}$. The following relations are convenient

$$
\begin{aligned}
& \gamma^{0} \frac{\left(1-\gamma_{5}\right)}{2}=\frac{\gamma^{0}+\gamma^{1}}{2} \equiv \gamma^{+}, \quad \gamma^{1} \frac{\left(1-\gamma_{5}\right)}{2}=\frac{\gamma^{1}+\gamma^{0}}{2}=\gamma^{+} \\
& V_{\mu} \gamma^{\mu} \frac{\left(1-\gamma_{5}\right)}{2}=\left(V_{0}+V_{1}\right)\left(\frac{\gamma^{0}+\gamma^{1}}{2}\right) \equiv V_{+} \gamma^{+} .
\end{aligned}
$$


Similarly

$$
\begin{aligned}
& \gamma^{0} \frac{\left(1+\gamma_{5}\right)}{2}=\frac{\gamma^{0}-\gamma^{1}}{2} \equiv \gamma^{-}, \quad \gamma^{1} \frac{\left(1+\gamma_{5}\right)}{2}=\frac{\gamma^{1}-\gamma^{0}}{2}=-\gamma^{-}, \\
& V_{\mu} \gamma^{\mu} \frac{\left(1+\gamma_{5}\right)}{2}=\left(V_{0}-V_{1}\right)\left(\frac{\gamma^{0}-\gamma^{1}}{2}\right) \equiv V_{-} \gamma^{-}, \\
& A_{ \pm}=A_{0} \pm A_{1}, \quad A^{ \pm}=\frac{1}{2}\left(A^{0} \pm A^{1}\right), \quad x_{ \pm}=x_{0} \pm x_{1}, \\
& \partial_{ \pm}=\partial_{0} \pm \partial_{1}, \quad \partial^{ \pm}=\frac{1}{2}\left(\partial^{0} \pm \partial^{1}\right)=\frac{1}{2}\left(\partial_{0} \mp \partial_{1}\right)=\frac{1}{2} \partial_{\mp}, \\
& A_{\mu} B^{\mu}=\eta^{+-} A_{+} B_{-}+\eta^{-+} A_{-} B_{+}, \quad \eta^{+-}=\eta^{-+}=\frac{1}{2}, \\
& \epsilon^{\mu \nu} A_{\mu} B_{\nu}=\epsilon^{+-} A_{+} B_{-}+\epsilon^{-+} A_{-} B_{+}, \quad \epsilon^{+-}=-\epsilon^{-+}=\frac{1}{2} .
\end{aligned}
$$

\section{B Proof of Cauchy's lemma}

We set

$$
f_{N}\left(x_{1}, x_{2}, \ldots, x_{N} ; y_{1}, y_{2}, \ldots, y_{N}\right) \equiv \operatorname{det}_{j, k}\left(\frac{1}{x_{j}-y_{k}}\right)
$$

and show that

$$
\begin{aligned}
& f_{N}\left(x_{1}, x_{2}, \ldots, x_{N} ; y_{1}, y_{2}, \ldots, y_{N}\right) \\
& =(-1)^{N(N-1) / 2} \frac{\prod_{j_{1}>j_{2}}^{N}\left(x_{j_{1}}-x_{j_{2}}\right) \prod_{k_{1}>k_{2}}^{N}\left(y_{k_{1}}-y_{k_{2}}\right)}{\prod_{j=1}^{N} \prod_{k=1}^{N}\left(x_{j}-y_{k}\right)}
\end{aligned}
$$

by mathematical induction.

It is easy to see that the assertion is true for $N=1$ (for this case, the numerator of the right-hand side of Eq. (55) is interpreted to be unity) and for $N=2$. Assuming that Eq. (55) is true when $N$ is replaced by $N-1$, we then show that it holds also for $N=N$.

It is obvious that, as a complex function of $x_{N}, f_{N}$ in Eq. (54) possesses simple poles at $y_{k}(k=1, \ldots, N)$. This property is shared also by the right-hand side of Eq. (55). Therefore, if the residues of both sides of Eq. (55) at the pole $x_{N}=y_{k}$ are the same, the difference of both sides is a bounded entire function of $x_{N}$ which (obviously) vanishes as $\left|x_{N}\right| \rightarrow \infty$. This implies the equality (55) by Liouville's theorem.

Now, from the definition (54), the residue of the left-hand side of Eq. (55) at the pole $x_{N}=y_{k}$ is given by the minor determinant, the determinant of the sub-matrix obtained by deleting the $N$-th row and $k$-th column. That is,

$$
(-1)^{N+k} f_{N-1}\left(x_{1}, x_{2}, \ldots, x_{N-1} ; y_{1}, \ldots, y_{k-1}, y_{k}, \ldots, y_{N}\right) .
$$

On the other hand, the residue of the right-hand side of Eq. (55) at the pole $x_{N}=y_{k}$ 
is

$$
\begin{aligned}
& \left.(-1)^{N(N-1) / 2} \frac{\prod_{j_{1}>j_{2}}^{N}\left(x_{j_{1}}-x_{j_{2}}\right) \prod_{k_{1}>k_{2}}^{N}\left(y_{k_{1}}-y_{k_{2}}\right)}{\prod_{j=1}^{N-1} \prod_{l=1}^{N}\left(x_{j}-y_{l}\right) \prod_{m \neq k}^{N}\left(x_{N}-y_{m}\right)}\right|_{x_{N}=y_{k}} \\
& =(-1)^{N(N-1) / 2} \frac{\prod_{n=1}^{N-1}\left(y_{k}-x_{n}\right) \prod_{j_{1}>j_{2}}^{N-1}\left(x_{j_{1}}-x_{j_{2}}\right) \prod_{k_{1}>k_{2}}^{N}\left(y_{k_{1}}-y_{k_{2}}\right)}{\prod_{j=1}^{N-1} \prod_{l=1}^{N}\left(x_{j}-y_{l}\right) \prod_{m \neq k}^{N}\left(y_{k}-y_{m}\right)} \\
& =(-1)^{N(N-1) / 2}(-1)^{N-1}(-1)^{N-k} \frac{\prod_{j_{1}>j_{2}}^{N-1}\left(x_{j_{1}}-x_{j_{2}}\right) \prod_{k_{1}>k>k_{2}}^{N}\left(y_{k_{1}}-y_{k_{2}}\right)}{\prod_{j=1}^{N-1} \prod_{l \neq k}^{N}\left(x_{j}-y_{l}\right)} .
\end{aligned}
$$

Assuming that Eq. (55) is valid when $N$ is replaced by $N-1$, it can be confirmed that

this last expression in Eq. (57) is equal to Eq. (56) and thus the residues of both sides of Eq. (55) are the same. By mathematical induction, this proves Eq. (55) for any $N$.

\section{Proof of an analogue of Cauchy's lemma for a bosonic spinor in Eq. (45)}

The rightmost expression of Eq. (45) is written as

$$
\begin{aligned}
& i^{N} f_{N}\left(\left(x_{1}\right)_{+},\left(x_{2}\right)_{+}, \ldots,\left(x_{N}\right)_{+} ;\left(y_{1}\right)_{+},\left(y_{2}\right)_{+}, \ldots,\left(y_{N}\right)_{+}\right) \\
& \quad \times i^{N} f_{N}\left(\left(y_{1}\right)_{-},\left(y_{2}\right)_{-}, \ldots,\left(y_{N}\right)_{-} ;\left(x_{1}\right)_{-},\left(x_{2}\right)_{-}, \ldots,\left(x_{N}\right)_{-}\right),
\end{aligned}
$$

if one introduces the function

$$
\begin{aligned}
& f_{N}\left(x_{1}, x_{2}, \ldots, x_{N} ; y_{1}, y_{2}, \ldots, y_{N}\right) \\
& \equiv \frac{1}{C_{N}\left(x_{1}, x_{2}, \ldots, x_{N} ; y_{1}, y_{2}, \ldots, y_{N}\right)}\left(\prod_{l=1}^{N} \frac{\partial}{\partial x_{l}}\right) C_{N}\left(x_{1}, x_{2}, \ldots, x_{N} ; y_{1}, y_{2}, \ldots, y_{N}\right),
\end{aligned}
$$

where

$$
C_{N}\left(x_{1}, x_{2}, \ldots, x_{N} ; y_{1}, y_{2}, \ldots, y_{N}\right) \equiv \prod_{j=1}^{N} \prod_{k=1}^{N} \frac{1}{x_{j}-y_{k}} \prod_{j_{1}>j_{2}}^{N}\left(x_{j_{1}}-x_{j_{2}}\right) .
$$

In what follows, we show that

$$
f_{N}\left(x_{1}, x_{2}, \ldots, x_{N} ; y_{1}, y_{2}, \ldots, y_{N}\right)=\sum_{\left\{k_{1}, k_{2}, \ldots, k_{N}\right\}} \frac{-1}{x_{1}-y_{k_{1}}} \frac{-1}{x_{2}-y_{k_{2}}} \cdots \frac{-1}{x_{N}-y_{k_{N}}}
$$

by mathematical induction. If this is true, then the rightmost term of Eq. (45) is

$$
\begin{aligned}
& {\left[\sum_{\left\{k_{1}, k_{2}, \ldots, k_{N}\right\}} \frac{1}{i\left(x_{1}-y_{k_{1}}\right)_{+}} \frac{1}{i\left(x_{2}-y_{k_{2}}\right)_{+}} \cdots \frac{1}{i\left(x_{N}-y_{k_{N}}\right)_{+}}\right]} \\
& \times\left[\sum_{\left\{k_{1}, k_{2}, \ldots, k_{N}\right\}} \frac{1}{i\left(y_{1}-x_{k_{1}}\right)_{-}} \frac{1}{i\left(y_{2}-x_{k_{2}}\right)_{-}} \cdots \frac{1}{i\left(y_{N}-x_{k_{N}}\right)_{-}}\right]
\end{aligned}
$$


and this coincides with the path integral over bosonic spinors on the left-hand side of Eq. (45).

It is easy to confirm that the assertion (61) is true for $N=1$ (for which the last factor in Eq. (60),$\prod_{j_{1}>j_{2}}^{N}\left(x_{j_{1}}-x_{j_{2}}\right)$, is interpreted as unity) and for $N=2$. Assuming that Eq. (61) is true when $N$ is replaced by $N-1$, we then show that it holds also for $N=N$.

From its definition in Eqs. (59) and (50), it is obvious that $f_{N}$, as the function of $x_{N}$, is a sum of simple poles possibly at $x_{j}(j \neq N)$ and $y_{k}$. Thus, to conclude Eq. (61), it suffices to determine the position of all poles as the function of $x_{N}$ and the residue at each pole.

Now we compute

$$
\begin{aligned}
& \frac{\partial}{\partial x_{N}} C_{N}\left(x_{1}, x_{2}, \ldots, x_{N} ; y_{1}, y_{2}, \ldots, y_{N}\right) \\
& =C_{N}\left(x_{1}, x_{2}, \ldots, x_{N} ; y_{1}, y_{2}, \ldots, y_{N}\right)\left(\sum_{k=1}^{N} \frac{-1}{x_{N}-y_{k}}+\sum_{j=1}^{N-1} \frac{1}{x_{N}-x_{j}}\right)
\end{aligned}
$$

and

$$
\begin{aligned}
& \frac{\partial}{\partial x_{N-1}} \frac{\partial}{\partial x_{N}} C_{N}\left(x_{1}, x_{2}, \ldots, x_{N} ; y_{1}, y_{2}, \ldots, y_{N}\right) \\
&=C_{N}\left(x_{1}, x_{2}, \ldots, x_{N} ; y_{1}, y_{2}, \ldots, y_{N}\right) \\
& \times {\left[\left(\frac{-1}{x_{N}-x_{N-1}}+\sum_{k=1}^{N} \frac{-1}{x_{N-1}-y_{k}}+\sum_{j=1}^{N-2} \frac{1}{x_{N-1}-x_{j}}\right)\right.} \\
& \times\left.\left(\frac{1}{x_{N}-x_{N-1}}+\sum_{n=1}^{N} \frac{-1}{x_{N}-y_{n}}+\sum_{m=1}^{N-2} \frac{1}{x_{N}-x_{m}}\right)+\frac{1}{\left(x_{N}-x_{N-1}\right)^{2}}\right] .
\end{aligned}
$$

First, examining the behavior of Eq. (64) near $x_{N} \sim x_{N-1}$, we immediately find that Eq. (64) is regular at $x_{N}=x_{N-1}$. Since in Eq. (159), further derivatives $\prod_{l=1}^{N-2} \partial / \partial x_{l}$ on Eq. (64) does not produce any singularity at $x_{N}=x_{N-1}$, we conclude that $f_{N}$ has no pole at $x_{N}=x_{N-1}$. Since the choice $x_{N-1}$ is of course arbitrary, we see that $f_{N}$ has no pole at $x_{j}$ as the function of $x_{N}$.

Next, from Eqs. (63) and (59), we see that the residue of the pole of $f_{N}$ at $x_{N}=y_{N}$ 
is given by

$$
\begin{gathered}
(-1)\left[\prod_{m=1}^{N-1} \prod_{n=1}^{N} \frac{1}{x_{m}-y_{n}} \prod_{m_{1}>m_{2}}^{N}\left(x_{m_{1}}-x_{m_{2}}\right)\right]^{-1} \\
\times\left.\left(\prod_{l=1}^{N-1} \frac{\partial}{\partial x_{l}}\right) \prod_{j=1}^{N-1} \prod_{k=1}^{N} \frac{1}{x_{j}-y_{k}} \prod_{j_{1}>j_{2}}^{N}\left(x_{j_{1}}-x_{j_{2}}\right)\right|_{x_{N}=y_{N}} \\
=(-1)\left[\prod_{m=1}^{N-1} \prod_{n=1}^{N-1} \frac{1}{x_{m}-y_{n}} \prod_{m_{1}>m_{2}}^{N-1}\left(x_{m_{1}}-x_{m_{2}}\right)\right]^{N-1} \\
\times\left(\prod_{l=1}^{N-1} \frac{\partial}{\partial x_{l}}\right) \prod_{j=1}^{N-1} \prod_{k=1}^{N-1} \frac{1}{x_{j}-y_{k}} \prod_{j_{1}>j_{2}}^{N-1}\left(x_{j_{1}}-x_{j_{2}}\right) \\
=-f_{N-1}\left(x_{1}, x_{2}, \ldots, x_{N-1} ; y_{1}, y_{2}, \ldots, y_{N-1}\right) .
\end{gathered}
$$

This is the function $f_{N}$ itself with one $N$ lower. The residue at the pole at $x_{N}=y_{k}$ for arbitrary $k$ is similarly obtained. Using this information and assuming that Eq. (61) with $N$ replaced by $N-1$ is valid, we see that Eq. (61) is true also for $N=N$. Eq. (61) holds for any $N$ by mathematical induction.

\section{Acknowledgments}

We thank Peter van Nieuwenhuizen for stimulating discussions on various aspects of quantum anomalies, which motivated the present work in particular. One of us (K.F.) thanks the hospitality at C. N. Yang Institute for Theoretical Physics where the present work was initiated. We also thank Henry Tye for asking the direct bosonization in path integral formulation. This work is supported in part by Japan Society for the Promotion of Science KAKENHI (Grant Nos. 25400415 and 23540330).

\section{References}

[1] S. R. Coleman, Phys. Rev. D 11, 2088 (1975).

[2] S. Mandelstam, Phys. Rev. D 11, 3026 (1975).

[3] A. M. Polyakov and P. B. Wiegmann, Phys. Lett. B 131, 121 (1983).

[4] A. M. Polyakov and P. B. Wiegmann, Phys. Lett. B 141, 223 (1984).

[5] E. Witten, Commun. Math. Phys. 92, 455 (1984).

[6] D. Gonzales and A. N. Redlich, Phys. Lett. B 147, 150 (1984).

[7] P. Di Vecchia and P. Rossi, Phys. Lett. B 140, 344 (1984). 
[8] J. Polchinski, Superstring theory and beyond, String Theory Vol. 2 (Cambridge University Press, Cambridge, 1998).

[9] M. Roček and P. van Nieuwenhuizen, Introduction to Modern String Theory (to be published).

[10] E. Abdalla, M. C. B. Abdalla and K. D. Rothe, Nonperturbative Methods in Twodimensional Quantum Field Theory (World Scientific, Singapore, 1991).

[11] M. Stone, Bosonization (World Scientific, Singapore, 1994).

[12] R. Roskies and F. Schaposnik, Phys. Rev. D 23, 558 (1981).

[13] R. E. Gamboa Saraví, F. A. Schaposnik and J. E. Solomin, Nucl. Phys. B 185, 239 (1981).

[14] K. Furuya, R. E. Gamboa Saraví and F. A. Schaposnik, Nucl. Phys. B 208, 159 (1982).

[15] C. M. Naón, Phys. Rev. D 31, 2035 (1985).

[16] K. Fujikawa and H. Suzuki, Path Integrals and Quantum Anomalies (Oxford University Press, Clarendon, 2004).

[17] R. Banerjee, Z. Phys. C 25, 251 (1984).

[18] M. Reuter and W. Dittrich, Phys. Rev. D 32, 513 (1985).

[19] J. N. Webb, Z. Phys. C 31, 301 (1986).

[20] A. K. Das and V. S. Mathur, Phys. Rev. D 33, 489 (1986).

[21] K. D. Rothe, Nucl. Phys. B 269, 269 (1986).

[22] R. Banerjee, Phys. Rev. Lett. 56, 1889 (1986).

[23] S. Miyake and K. Shizuya, Phys. Rev. D 36, 3781 (1987).

[24] S. Miyake and K. Shizuya, Phys. Rev. D 37, 2282 (1988).

[25] M. N. Sanielevici, G. W. Semenoff and Y.-S. Wu, Nucl. Phys. B 312, 197 (1989).

[26] K. Shizuya, Phys. Lett. B 213, 298 (1988).

[27] X.-Q. Luo and Q.-Z. Chen, J. Phys. G 16, 1181 (1990).

[28] C. M. Naón, M. C. von Reichenbach and M. L. Trobo, Nucl. Phys. B 435, 567 (1995) hep-th/9409085.

[29] N. Banerjee and R. Banerjee, Nucl. Phys. B 445, 516 (1995). 
[30] J. V. Steele, A. Subramanian and I. Zahed, Nucl. Phys. B 452, 545 (1995) hep-th/9503220.

[31] D. J. Gross, I. R. Klebanov, A. V. Matytsin and A. V. Smilga, Nucl. Phys. B 461, 109 (1996) hep-th/9511104.

[32] S. D. Joglekar and G. Saini, Phys. Rev. D 43, 1355 (1991).

[33] E. M. C. Abreu, R. Banerjee and C. Wotzasek, Nucl. Phys. B 509, 519 (1998) hep-th/9707204.

[34] M. Faber and A. N. Ivanov, Eur. Phys. J. C 20, 723 (2001) hep-th/0105057.

[35] K. Fujikawa and H. Suzuki, Phys. Rept. 398, 221 (2004) hep-th/0305008.

[36] G. Grignani, D. Marmottini and P. Sodano, Mod. Phys. Lett. A 19, 2485 (2004) hep-lat/0310062.

[37] A. Iucci and C. Naón, J. Phys. A 38, 749 (2005) [hep-th/0311128].

[38] C. M. Naón, M. J. Salvay and M. L. Trobo, Int. J. Mod. Phys. A 19, 4953 (2004) hep-th/0312142.

[39] H. Bozkaya, A. N. Ivanov and M. Pitschmann, J. Phys. A 39, 11075 (2006) hep-th/0512286.

[40] T. Radożycki, Eur. Phys. J. C 55, 509 (2008) arXiv:0801.4399 [hep-th]].

[41] Y. C. Kao and M. C. Wu, Phys. Rev. D 85, 027701 (2012) [arXiv:1104.4373 [hep-th]].

[42] E. Fradkin, E. F. Moreno and F. A. Schaposnik, Phys. Lett. B 730, 284 (2014) arXiv:1401.3018 [hep-th]].

[43] R. E. G. Saraví, C. M. Naón and F. A. Schaposnik, J. Stat. Mech. 1409, no. 9, P09034 (2014) [arXiv:1406.4578 [hep-th]].

[44] D. N. Blaschke, R. Carballo-Rubio and E. Mottola, JHEP 1412, 153 (2014) arXiv:1407.8523 [hep-th]].

[45] R. Bufalo and B. M. Pimentel, Int. J. Mod. Phys. A 29, 1450122 (2014) arXiv:1408.2917 [hep-th]].

[46] K. Fujikawa, Phys. Rev. Lett. 42, 1195 (1979).

K. Fujikawa, Phys. Rev. D 21, 2848 (1980) [Erratum-ibid. D 22, 1499 (1980)].

[47] D. Friedan, E. J. Martinec and S. H. Shenker, Nucl. Phys. B 271, 93 (1986).

[48] K. Fujikawa, Phys. Rev. D 25, 2584 (1982).

[49] A. M. Polyakov, Phys. Lett. B 103, 207 (1981). 
[50] O. Alvarez, Nucl. Phys. B 216, 125 (1983).

[51] P. Bouwknegt and P. van Nieuwenhuizen, Class. Quant. Grav. 3, 207 (1986).

[52] M. Roček, P. van Nieuwenhuizen and S. C. Zhang, Annals Phys. 172, 348 (1986). 